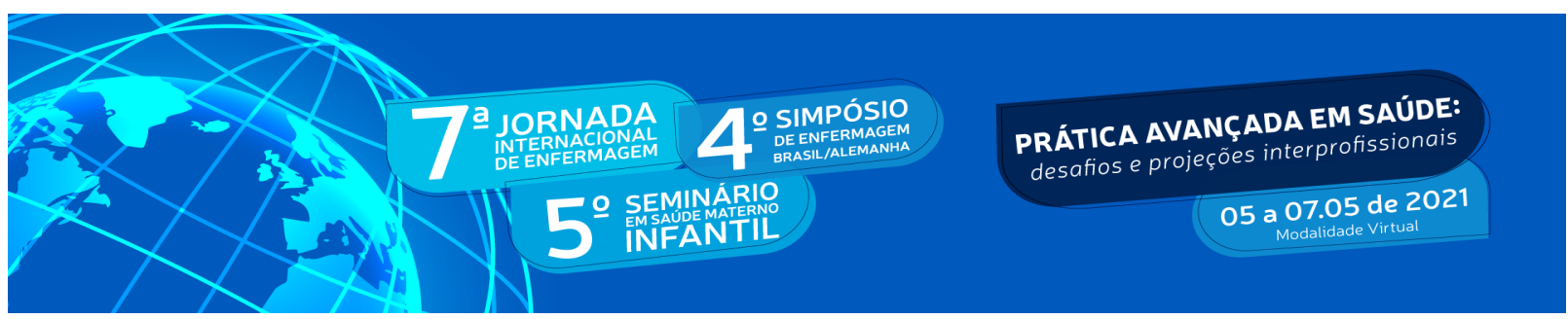

DOI: http://doi.org/10.48195/jie2021-123

\title{
PADRÃO ALIMENTAR DE CRIANÇAS MAIORES DE SEIS ANOS
}

\section{Ana Paula Gularte Barbosa'; Marcia Montagner²; Michele Vargas Reginatto ${ }^{3}$; Júlia Schmitt Neves ${ }^{4}$; Franceliane Jobim Benedetti ${ }^{5}$}

\begin{abstract}
RESUMO
O objetivo da presente pesquisa foi identificar na literatura o consumo de alimentos industrializados e o quanto eles influenciam no padrão alimentar das crianças. Foi realizada uma revisão narrativa da literatura, nos meses de maio a junho de 2018, em periódicos da área da saúde. Os estudos avaliados revelaram que a alimentação das crianças apresenta alto consumo de gorduras e açúcares, provenientes dos alimentos industrializados, responsáveis pelo aumento da obesidade infantil. Conclui-se que o padrão alimentar das crianças brasileiras está aquém do desejado e, para que ele possa melhorar, precisa maior ingestão de alimentos in natura e minimamente processados e menor de ultraprocessados
\end{abstract}

Palavras-chave: Alimentos industrializados; Consumo de alimentos; Crianças.

\begin{abstract}
The aim of this research was to identify in the literature the consumption of processed foods and how much they influence the eating patterns of children. A narrative review of the literature was carried out from May to June 2018, in healthcare journals. The evaluated studies revealed that children's diets have a high consumption of fats and sugars, derived from processed foods, which are responsible for the increase in childhood obesity. It is concluded that the dietary pattern of Brazilian children is below the desired level and, in order for it to improve, it needs a higher consumption of fresh and minimally processed foods and a lower level of ultra-processed foods.
\end{abstract}

Keywords: Processed foods; Food consumption; Children.

\footnotetext{
${ }^{1}$ Ma. em Saúde Materno Infantil.Universidade Franciscana- UFN. E-mail: ana.gularte@ufn.edu.br

${ }^{2}$ Ma. em Saúde Materno Infantil.Universidade Franciscana- UFN. E-mail: marcia.montagner@ufn..edu.br

${ }^{3}$ Ma. em Saúde Materno Infantil.Universidade Franciscana- UFN. E-mail: mi.reginatto@gmail.com

${ }^{4}$ Graduanda de Nutrição e bolsista PET- Saúde-interprofssionalidade. Universidade Franciscana- UFN. E-mail: juliaaschmitt@outlook.com

${ }_{5}^{5}$ Prof ${ }^{a}$. Dra. do Curso de Nutrição e Mestrado Profissional Materno Infantil. Universidade Franciscana- UFN. E-mail:franceliane@ufn.edu.br
} 


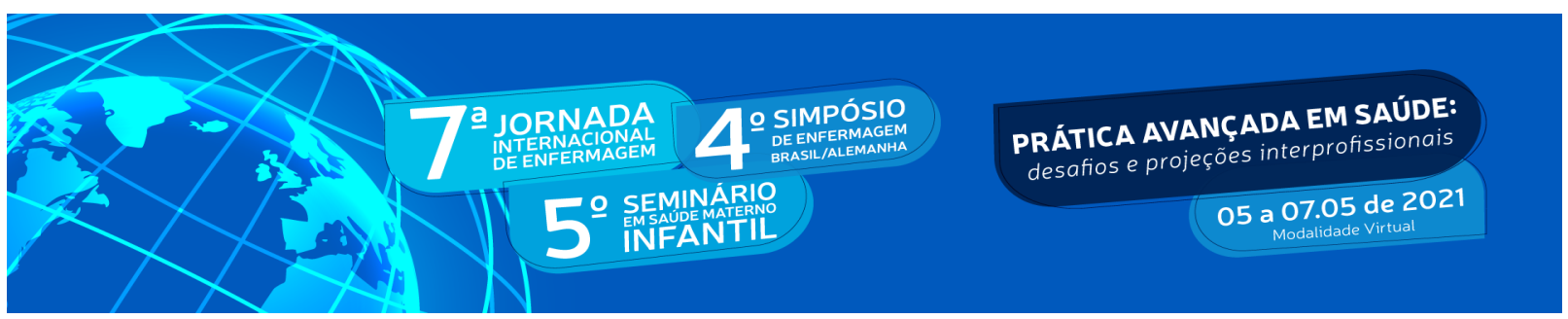

\section{INTRODUÇÃO}

A alimentação e nutrição consistem em condições básicas para a melhoria e a proteção da saúde, possibilitando a afirmação plena do potencial de crescimento e progresso humano, com qualidade de vida e cidadania (BRASIL, 2012).

O consumo alimentar da criança é influenciado pela família, pela escola e pelos amigos e as alterações do hábito alimentar são apontadas como fatores causais do aumento progressivo da obesidade infantil (RINALDI et al., 2008). Isto, em virtude do aumento do consumo de alimentos industrializados, que são os processados e ultraprocessados, veiculados, diariamente, pela mídia, financiada por grandes empresas alimentícias (MATUK, et al, 2011).

O padrão alimentar da população brasileira é fortemente caracterizado por uma combinação de uma dieta dita "tradicional" (arroz com feijão), conforme Guia Alimentar para a População Brasileira (BRASIL, 2014), são categorizados em alimentos minimamente processados ou in natura que, antes de sua aquisição, foram submetidos a alterações mínimas, como secagem, lavagem, moagem entre outras e, o mesmo tempo, rica em alimentos com alto teor calórico (BRASIL, 2012), classificados em ultraprocessados, que são alimentos com alta concentrações de gorduras, sódio, açúcar e com baixo teor de micronutrientes (vitaminas e minerais) (BRASIL, 2014).

Em relação ao consumo de frutas, legumes e verduras (FLV), classificados pelo Guia Alimentar para a População Brasileira (BRASIL, 2014), como alimentos in natura, encontram-se estável na última década, mas, ainda, é metade do recomendado pela Organização Mundial da Saúde (OMS) e Organização das Nações Unidas para a Alimentação e a Agricultura (FAO), mínimo de cinco porções ao dia, pelo menos cinco vezes na semana, pois são ricos em fibras, vitaminas e minerais. que auxiliam na prevenção de Doenças Crônicas Não Transmissíveis (DCNT) (BRASIL, 2014).

A alimentação infantil, quando inadequada, composta, frequentemente, por alimentos industrializados pode contribuir para o aumento do risco de sobrepeso e obesidade (CHUNG et al. 2018). A incidência da obesidade infantil no Brasil já é uma realidade, um terço das crianças com idades entre cinco e nove anos está acima do peso (IBGE, 2010). Nesse sentido, 


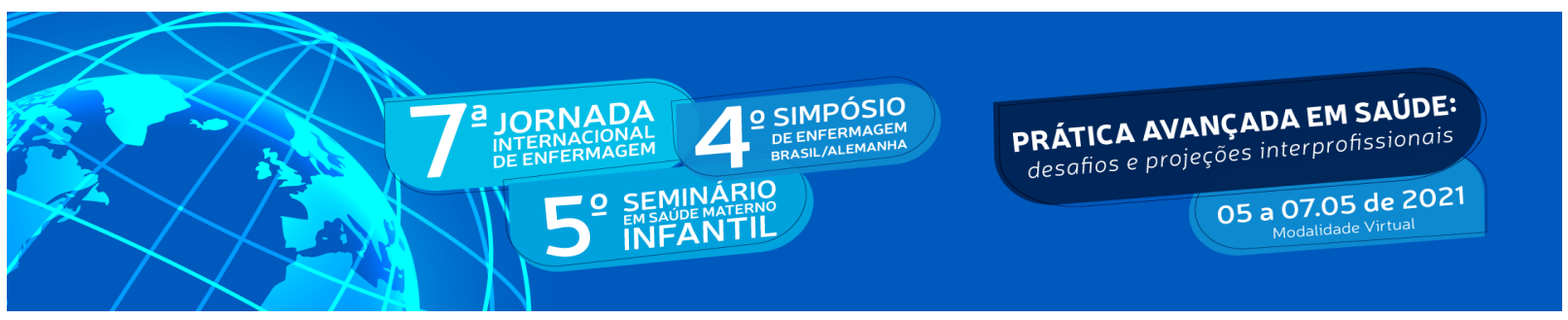

o Guia Alimentar para População Brasileira (BRASIL, 2014), é um importante instrumento de apoio e incentivo às práticas alimentares saudáveis para a prevenção de DCNT, uma vez que fornece recomendações sobre a escolha de alimentos, de acordo com o tipo de processamento empregado na sua produção.

\section{OBJETIVO}

Identificar o consumo de alimentos industrializados e o quanto eles influenciam no padrão alimentar das crianças .

\section{METODOLOGIA}

Realizou-se uma revisão narrativa da literatura, nos meses de maio a junho de 2018, nos periódicos: Portal Regional da BVS, National Library of Medicine (PubMed), Science Direct, Scientific Electronic Library Online (SciELO) e Literatura Latino-Americana em Ciências da Saúde (Lilacs), com o propósito de identificar o consumo de alimentos industrializados e o quanto eles influenciam no padrão alimentar das crianças.

Utilizou-se os seguintes descritores em português, inglês e espanhol: "Consumo de alimentos" (Food consumptions, consumo de alimentos), "Alimentos industrializados" (industrialized foods, alimentos industrializados) e o operador booleano "AND". A seleção seguiu os seguintes critérios de inclusão: não foi delineado o ano das publicações; texto completo para o acesso; idiomas em inglês, português e espanhol; que apresentassem como foco do estudo a investigação sobre o consumo dos alimentos industrializados por crianças e, excluídos os artigos que pesquisaram consumo de alimentos industrializados de crianças menores de 6 anos, adolescentes, adultos e idosos.

\section{RESULTADOS E DISCUSSÃO}

Dos estudos encontrados, destaca-se o alto consumo de gordura, de açúcares e de outros produtos industrializados, entre as crianças. Em Kersting et al. (1992), constatou-se 


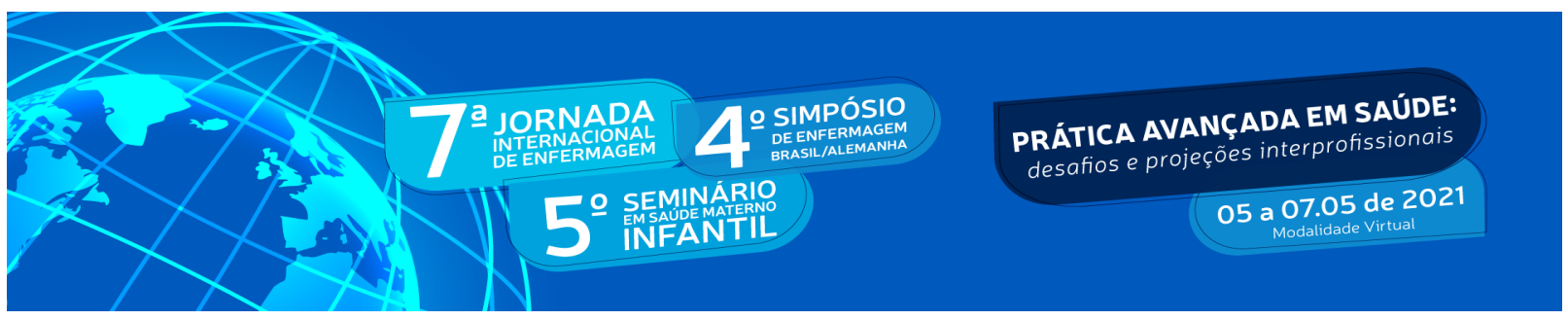

que as crianças ingeriam uma grande quantidade de refrigerantes e chás adoçados. Em Sangalli; Rouber; Vitolo (2016), o consumo de alimentos saudáveis entre crianças, encontra-se abaixo das recomendações, com exceção do feijão e mais da metade das crianças consomem alimentos ultraprocessados, consequentemente, inadequação na ingestão de micronutrientes.

Outros estudos, apontaram menor ingestão de fibras, encontradas em FLV, com grande poder de saciedade (BRASIL, 2014), entre crianças que consomem processados e ultraprocessados (BARCELOS et al., 2014; LOUZADA et al., 2015; ARES et al., 2016; VEGA et al., 2015; ENES; SLATER, 2010), como indica Sparrenbergeret et al. (2015), em que metade dos alimentos ingeridos pelas crianças são ultraprocessados e a inadequação alimentar pode levar ao desenvolvimento precoce de sobrepeso e obesidade, além de outras doenças crônicas associadas (TRICHES; GIUGLIANI, 2005).

De acordo com o Guia Alimentar para a População Brasileira (BRASIL, 2014), os alimentos in natura ou minimamente processados são submetidos a processos de limpeza, secagem, retiradas de talos, cascas e etc. sem adição de produtos químicos. Já os processados e ultraprocessados, além de serem fabricados com excesso de açúcares e gorduras saturadas e trans, usam, exageradamente, ingredientes diferentes, que não fazem parte do processo de preparação dos alimentos da culinária familiar habitual (BRASIL, 2014).

Souza et al. (2017), verificou elevado consumo de industrializados, fontes de proteína animal, gorduras e açúcares refinados e baixo consumo de fibras e pescados. Estudo que traça o perfil nutricional do consumo de alimentos industrializados dos brasileiros, mostrou que a maior densidade energética se deu pelo consumo de gorduras saturadas e trans e altos níveis de açúcar livre e menor ingestão de fibras, quando comparado com a fração de consumo de alimentos minimamente processados (LOUZADA et al., 2015). A inclusão de alimentos ultraprocessados na dieta resultou em deterioração geral do perfil nutricional da dieta em todas as faixas etárias, incluindo as crianças (BARCELOS et al., 2014).

\section{CONCLUSÃO}

Conclui-se que a qualidade do padrão alimentar das crianças encontra-se comprometida, 


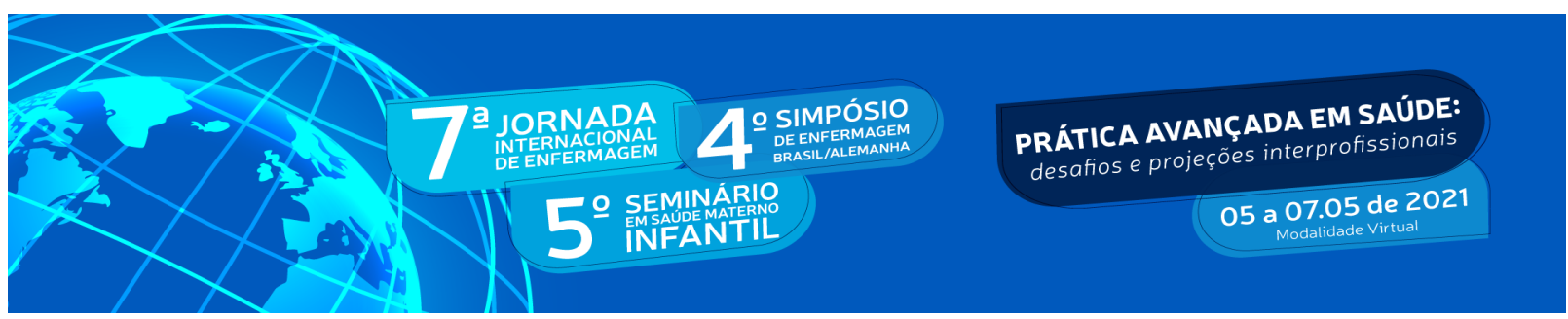

já que há uma elevada ingestão de alimentos industrializados, que podem aumentar o risco de desenvolver doenças como a obesidade, ainda, na infância. E, para que o padrão alimentar das crianças melhore, deve-se aumentar o consumo de alimentos in natura e minimamente processados.

\section{REFERÊNCIAS}

ANZOLIN, C. et al. Intervenções nutricionais em escolares. Revista Brasileira em Promoção da Saúde, v. 23, n. 4, p. 297-306, 2010. Disponível em: https://periodicos.unifor.br/RBPS/article/view/2030. Acesso em: 08 mar. 2021.

ARES, G. et al. Consumers' conceptualization of ultra-processed foods. Appetite, v. 105, p. 611-617, 2016. Disponível em: https://doi.org/10.1016/j.appet.2016.06.028. Acesso em: 08 mar. 2021.

BARCELOS, G. T., RAUBER, F., VITOLO, M. R. Processed and ultra-processed food products and nutrient intake in children. Ciência \& Saúde, v. 7, n. 3, 155-161, 2014.

Disponível em: https://doi.org/10.15448/1983-652X.2014.3.19755. Acesso em: 08 mar. 2021.

BRASIL. Ministério da Saúde. Secretaria de Atenção à Saúde. Departamento de Atenção Básica. Guia alimentar para a população brasileira. 2. ed., 1. reimpr. Brasília: Ministério da Saúde, 2014. Disponível em:

https://bvsms.saude.gov.br/bvs/publicacoes/guia_alimentar_populacao_brasileira_2ed.pdf . Acesso: 08 mar. 2021.

BRASIL. Ministério da Saúde. Secretaria de Atenção à Saúde. Departamento de Atenção Básica. Política Nacional de Alimentação e Nutrição. 1. ed., 1. reimpr. Brasília: Ministério da Saúde, 2013. Disponível em:

https://bvsms.saude.gov.br/bvs/publicacoes/politica_nacional_alimentacao_nutricao.pdf. Acesso: 08 mar. 2021.

CHUNG, A. et al. Contribution of discretionary food and drink consumption to socio-economic inequalities in children's weight: prospective study of Australian children. International Journal of Epidemiology, v. 47, n. 3, p. 820-828, 2018. Disponível em: https://doi.org/10.1093/ije/dyy020. Acesso em: 08 mar. 2021.

ENES, C. C.; SLATER, B. Obesidade na adolescência e seus principais fatores determinantes. Revista Brasileira de Epidemiologia, v. 3, n. 1, p. 163-171, 2010. Disponível em: http://dx.doi.org/10.1590/S1415-790X2010000100015. Acesso: 08 mar. 2021.

IBGE - Instituto Brasileiro de Geografia e Estatística. Ministério do Planejamento, Orçamento e Gestão. Coordenação de Trabalho e Rendimento. Pesquisa de Orçamentos Familiares 2008-2009: antropometria e estado nutricional de crianças, adolescentes e adultos no Brasil. 


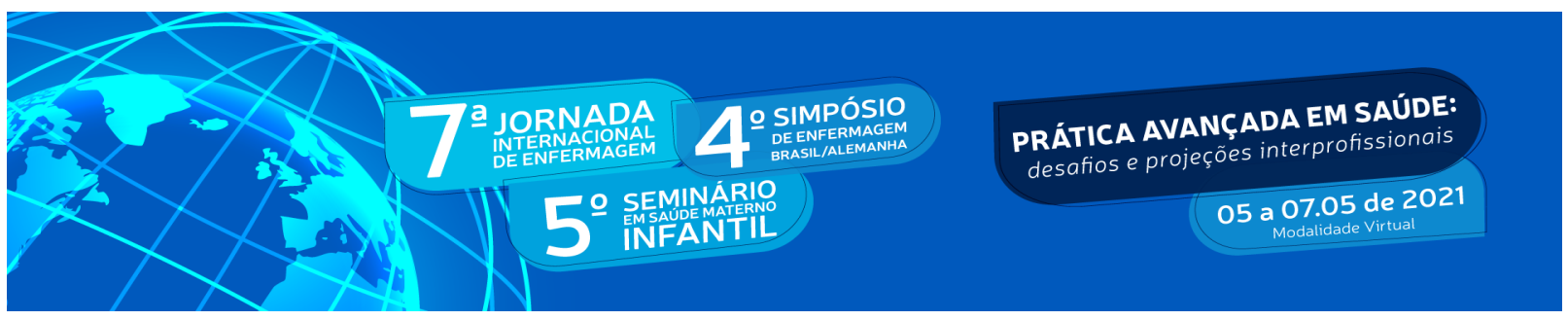

Rio de Janeiro: Ministério do Planejamento, Orçamento e Gestão, 2010. Disponível em: https://biblioteca.ibge.gov.br/visualizacao/livros/liv45419.pdf. Acesso: 08 mar. 2021.

KERSTING, M.; TROPH, D.; SCHÖCH, G. Achievable guidelines for food consumption to reach a balanced fat and nutrient intake in childhood and adolescence. Journal of the American College of Nutrition, v. 11, sup. 1, p. 74S-78S, 1992. Disponível em: https://doi.org/10.1080/07315724.1992.10737989. Acesso em: 08 mar. 2021.

LOUZADA, M. L. D. C. et al. Ultra-processed foods and the nutritional dietary profile in Brazil. Revista de Saúde Pública, v. 49, 2015. Disponível em:

https://doi.org/10.4000/confins.18449. Acesso em: 08 mar. 2021.

MATUK, T. T. et al. Composição de lancheiras de alunos de escolas particulares de São Paulo. Revista Paulista de Pediatria, v. 29, n. 2, p. 157-163, 2011. Disponível em: https://doi.org/10.1590/S0103-05822011000200005. Acesso em: 08 mar. 2021.

RINALDI, A. E. M. et al. Contribuições das práticas alimentares e inatividade física para o excesso de peso infantil. Revista Paulista de Pediatria, vol. 26, n. 3, p. 271-277, 2008. Disponível em: https://doi.org/10.1590/S0103-05822008000300012. Acesso em: 08 mar. 2021.

ROSSI, A.; MOREIRA, E. A. M.; RAUEN, M. S. Determinantes do comportamento alimentar: uma revisão com enfoque na família. Revista de Nutrição, v. 21, n. 6, p. 739-748, 2008. Disponível em: https://doi.org/10.1590/S1415-52732008000600012. Acesso em: 08 mar. 2021.

SANGALLI, C. N.; RAUBER, F.; VITOLO, M. R. Low prevalence of inadequate micronutrient intake in young children in the south of Brazil: a new perspective. British Journal of Nutrition, v. 116, n. 5, p. 890-896, 2016. Disponível em: 10.1017 / S0007114516002695. Acesso em: 08 mar. 2021.

SOUZA, C. D. S. M. et al. Consumo alimentar de crianças do ensino fundamental em uma instituição pública. Revista Baiana de Enfermagem, v. 31, n. 2, 2017. Disponível em: http://dx.doi.org/10.18471/rbe.v31i2.20583. Acesso em: 08 mar. 2021.

SPARRENBERGER, K. et al. Ultra-processed food consumption in children from a Basic Health Unit. Jornal de pediatria, v. 91, n. 6, p. 535-542, 2015. Disponível em: https://doi.org/10.1016/j.jped.2015.01.007. Acesso em: 08 mar. 2021.

TRICHES, R. M.; GIUGLIANI, E. R. J. Obesidade, práticas alimentares e conhecimentos de nutrição em escolares. Revista de Saúde Pública, v. 39, p. 541-547, 2005. Disponível em: https://doi.org/10.1590/S0034-89102005000400004. Acesso em: 08 mar. 2021.

VEGA, J. B.; POBLACION, A. P.; TADDEI, J. A. A. C. Fatores associados ao consumo de bebidas açucaradas entre pré-escolares brasileiros: inquérito nacional de 2006. Ciência \& Saúde Coletiva, 20, 2371-2380, 2015. Disponível em: https://doi.org/10.1590/1413-81232015208.18032014. Acesso em: 08 mar. 2021. 